
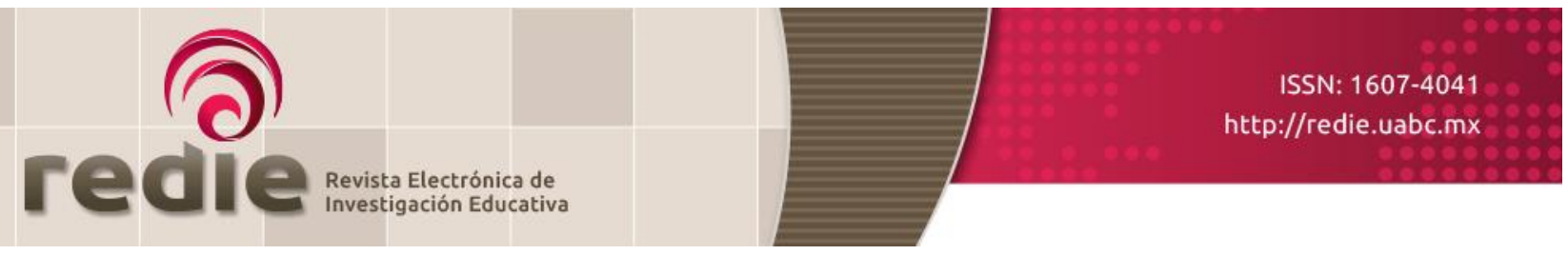

Vol. 20, Núm. 3, 2018

\title{
Redes educativas locales para la mejora escolar
}

\section{Local Education Networks for School Improvement}

\author{
Elena Hernández de la Torre (*) eht@us.es \\ María José Navarro Montaño (*) \\ (*) Universidad de Sevilla \\ (Recibido: 24 de octubre de 2016; Aceptado para su publicación: 6 de marzo de 2017)
}

Cómo citar: Hernández, E. y Navarro, M. J. (2018). Redes educativas locales para la mejora escolar. Revista Electrónica de Investigación Educativa, 20(3), 29-42. https://doi.org/10.24320/redie.2018.20.3.1669

\section{Resumen}

Este estudio indaga en el funcionamiento, dilemas y controversias de centros que trabajan en redes educativas locales de forma cooperativa con el objetivo de desarrollar procesos de planificación, gestión y formación conjunta, partiendo de un trabajo colaborativo que propicie el progreso y la mejora de los centros. Рara ello se utilizaron estrategias de recogida de datos tanto cualitativo como cuantitativo, cuestionarios y técnicas biográfico-narrativas a profesionales implicados en el trabajo de las redes escolares: entrevistas a asesores de zona, coordinadores de redes escolares, observaciones y grabaciones de sesiones de trabajo en grupo y cuestionarios a directores de centros y profesorado participante. Los resultados indican que el trabajo en redes escolares propicia la creación de una estructura organizativa inter-centros, el reparto de responsabilidades, aprendizajes en colaboración, el establecimiento de consenso entre los centros y el incremento de una filosofía de trabajo basada en la equidad, aprendizaje para todos y mejora escolar.

Palabras clave: Aprendizaje colaborativo, innovación, redes escolares, trabajo en equipo.

\section{Abstract}

This study explores the operation of, and predicaments and controversies in, schools that work together in local education networks to develop planning, management, and joint training processes based on collaborative work that fosters progress and improvement in schools. This was done using qualitative and quantitative data collection strategies, questionnaires and biographical-narrative techniques with professionals working in school networks: interviews with district advisors, education network coordinators, observations and recordings from working group sessions, and questionnaires administered mostly to school principals and teachers. The results show that working in education networks promotes the creation of an inter-school organizational structure, responsibility sharing, collaborative learning, consensus-building between schools, and an increase in a work philosophy based on equity, learning for all, and school improvement.

Keywords: Collaborative learning, innovation, school networks, teamwork. 


\section{Introducción}

El desarrollo profesional en redes constituye actualmente una forma de trabajo eficaz e importante para el ser humano, tanto a nivel social en general como en los centros educativos en particular, es este el tema que desarrollamos aquí. Las redes educativas se definen como estructuras intencionales, con intereses-objetivos comunes en las que todos sus miembros tienen la posibilidad de trabajar y responsabilizarse en igualdad. Esto genera una gran cohesión de grupo que incrementa la confianza, así como un fortalecimiento de relaciones satisfactorias (Gobierno Vasco, 2011, p. 4).

Investigaciones recientes acerca del funcionamiento de las redes educativas locales escolares en España (Civís y Longás, 2015; Muñoz, 2010) señalan que éstas son estructuras organizativas de carácter interescolar que ofrecen la oportunidad de aprender a los profesores que en ellas participan a partir del estudio a fondo de las necesidades de sus propios centros escolares. Pertenecer a redes educativas escolares parece favorecer este análisis de necesidades y el funcionamiento de cada centro, son un medio idóneo para abordar nuevas propuestas de trabajo y desarrollar responsabilidades compartidas para atender a la diversidad del alumnado en todos los ámbitos.

En este sentido se ha observado en el trabajo de las redes escolares que los centros, a medida que se incorporan a la red escolar, desarrollan distintas funciones en la red, dando lugar a lo que se denomina liderazgo distribuido en la innovación escolar (Vilar, 2008), considerándose la piedra angular de los Proyectos de Mejora y Planes de Atención a la Diversidad. Los cambios que se requieren en las escuelas hoy día necesitan un equilibrio entre motivación, apoyo del grupo y trabajo en red compartido, abordando una estrategia planificada por todos, es decir, "las redes son una buena alternativa para integrar esfuerzos, recursos y planes conjuntos" (Gobierno Vasco, 2011, p. 5).

La finalidad que se persigue hoy en día para la mejora educativa con la constitución de estas redes de centros educativos es la creación de experiencias innovadoras en los centros, la coordinación estable y duradera de los centros participantes para constituir una red de comunicación estable y el traslado de experiencias e información de unos centros a otros. Estas redes se denominan actualmente "redes escolares efectivas para mejorar la eficiencia y la calidad de los centros participantes" (Ministerio de Educación del Salvador, 2004-2009).

Los resultados de estudios acerca del trabajo de los centros escolares en red (Elboj, Puigdellivol, Soler y Valls, 2002; Hadfield y Chapman, 2009) señalan que esta forma de trabajar tiene la característica de crear una coordinación estable de centros con el propósito de constituir una red de experiencias de unos centros a otros. Se trata de construir una red de intercambio, de relación y cooperación, generadoras de procesos innovadores y participativos de los profesores que comparten el proceso educativo. Esta dimensión social de la red elabora un sistema de relaciones entre centros y profesorado con el fin de que los cambios sean colectivos (Muñoz, 2010), con la participación de todos los profesores implicados, y considerando que este cambio puede ser lento y progresivo, pero sobre todo formativo.

Este proceso de participación conlleva unas relaciones de trabajo entre profesorado para el desarrollo de una cultura colaborativa. La literatura señala que una de las ventajas del trabajo en red es que ayuda al profesorado a superar el síndrome de agotamiento o burnout como consecuencia de las barreras pedagógicas, didácticas y organizativas de la enseñanza (Betoret y Artiga, 2010) encontrando que éste se considera una barrera percibida por el profesorado como la peor de las causas que frenan la innovación y el cambio en los centros educativos.

El trabajo en red local, señalan estas investigaciones, parece que ayuda a diseñar nuevos procesos de trabajo basados en el desarrollo comunitario, se trata de un "acompañamiento en el trabajo", de un proyecto compartido donde el profesorado planifica para la práctica y representa el modo en que un centro responde a sus necesidades de atención al alumnado y desarrolla formas colaborativas de trabajo. Una de las conclusiones que se extrae de esta forma de trabajo es la interdependencia entre el profesorado para trabajar y, como consecuencia, las escuelas mejoran en el estudio de sus propias necesidades, en el uso de estrategias didácticas y organizativas, en las evaluaciones del alumnado, etc. 
Este estudio pone de manifiesto resultados y reflexiones compartidas con otros investigadores españoles, como Betoret y Artiga (2010), Civís y Longás (2015), Doménech (2005), Elboj, Puigdellivol, Soler y Valls (2002), Muñoz (2010), Rué (2005) y Rué et al. (2005); así como con estudios internacionales como los de Ainscow y West (2006), Ainscow, Muijs y West (2006), Chapman (2006), Chapman y Fullan (2007), Dufour, Eaker y Many (2006), Hadfield y Chapman (2009) y Wohlstetter y Smith (2000).

Los estudios de redes locales, la "formación en red" y el conocimiento compartido para trabajar mejor abordan mejoras e innovaciones en centros con un grupo de trabajo amplio, paliando con ello el trabajo en aislamiento de cada centro docente y de su profesorado. Debido a ello planteamos analizar los procesos de reflexión conjunta que se abordan tanto en la red como en cada centro partiendo de las aportaciones de investigaciones anteriores.

La hipótesis de nuestro estudio plantea que el trabajo en redes educativas locales supone en la actualidad una necesidad para el cambio en los centros y para la mejora, donde la finalidad de cada centro es optimizar la calidad educativa que necesitan para responder a las nuevas demandas de los centros educativos actuales y para el desarrollo de una educación compartida, de calidad y de responsabilidad de todos. Esto se consigue a través del trabajo conjunto, con aportaciones de la comunidad educativa y cambios consensuados en el entorno escolar, basado en el contexto y desarrollados a partir de él.

El trabajo en la red, por tanto, se define por un enfoque horizontal y colectivo que promueve la reflexión y el intercambio entre todos los participantes. Las relaciones que se establecen entre sus miembros son recíprocas, personalmente significativas y sobre todo formativas, llegando a acuerdos de funcionamiento entre los miembros que la componen. Estas relaciones se basan en el respeto y la escucha mutua, con lo que la comunidad genera contextos para el crecimiento del profesorado y del grupo, cimentados en el respeto por la independencia y el fomento de la interdependencia. Estos estudios se encuentran relacionados con los de las escuelas como comunidades democráticas, deliberativas y de aprendizaje entre los propios centros educativos.

Basamos este estudio en las afirmaciones de Bolívar (2015), quien plantea la confianza en el desarrollo de la escuela como una comunidad profesional:

Perdida la confianza en los cambios planificados externamente para mejorar por sí solos la educación, actualmente se apuesta por movilizar localmente la capacidad interna de cambio de las escuelas como organizaciones, individuos y grupos para regenerar internamente la mejora de la educación (Bolívar, 2015, p. 1)

En el estudio se trabaja con dos redes educativas de centros escolares con el propósito de analizar el funcionamiento, dilemas y controversias que se suscitan en su puesta en marcha y desarrollo. En la Red A nos centramos en educación primaria y en la Red B en educación secundaria.

\section{Método}

El propósito de este estudio es indagar en la planificación y gestión del trabajo colaborativo en dos redes educativas (primaria y secundaria) analizar el trabajo conjunto que realizan y cuál es su repercusión para la innovación en los centros educativos. A partir de aquí se plantean las siguientes cuestiones tomando en cuenta entrevistas y cuestionarios: inicio del trabajo y formación de cada red, diseño y desarrollo de la RED, recursos, organización del trabajo en red, repercusiones en los centros y en la comunidad educativa, impacto de la participación en la red en los centros educativos y resultados.

A partir de esta información se explica el funcionamiento y desarrollo de dichas redes, se valora su aportación a los centros pertenecientes a la misma, y se analiza la respuesta del profesorado a partir de su pertenencia a la red educativa y los dilemas que se generan. Se recogieron datos de todos los miembros de la comunidad educativa de las redes (profesorado, equipos directivos, asesores y coordinadores) a través de cuestionarios semiestructurados individuales y entrevistas semiestructuradas de tipo comprehensivo, partiendo de una metodología descriptivo-narrativa fundamentada en un enfoque cualitativo y cuantitativo de investigación. El análisis de los cuestionarios semiestructurados se 
realizó con el programa estadístico SPSS 22.0 considerando por una parte el recuento a nivel de frecuencias y porcentajes de respuestas (preguntas cerradas); para el análisis de las preguntas abiertas y entrevistas se empleó el programa de análisis cualitativo MAXQDA 10.

La metodología utilizada fue de corte participativo y colaborativo, interactuando y contactando continuamente con las redes en reuniones individuales y de grupo (entrevistas focales o focus group y entrevistas individuales). Respecto a los participantes/informantes de las dos redes estudiadas, éstos han sido asesores de Centros de Profesorado, profesorado de los centros, equipos directivos, orientadores de centros y coordinadores de Grupos de Trabajo, así como inspectores de zona. El proceso de recogida de datos y las fases estructuraron de la siguiente forma:

Tabla I. Fases del proceso de recogida de datos (período 2012-2015)

\begin{tabular}{|c|c|c|}
\hline FASES & Proceso de Recogida de Datos & Población/Participantes \\
\hline $\begin{array}{l}\text { Primera: } \\
\text { Estudio de necesidades } \\
\text { ( } 3 \text { meses) }\end{array}$ & $\begin{array}{l}\text { Contacto por email con } 6 \text { CEPs-Centros de } \\
\text { Profesorado) (cita) } \\
\text { Cuestionario estructurado asesores ( } 7 \text { ítem) } \\
\text { Entrevistas estructuradas en grupos focales } \\
\text { ( } 6 \text { ítem) }\end{array}$ & $\begin{array}{l}\text { Carta informativa del Proyecto Redes } \\
\text { Cuestionario asesores de centros de profesores } \\
\text { Entrevistas focales a asesores/ directores de } \\
\text { Centros de Profesorado (10 asesores de } 3 \text { CEPs- } \\
\text { Centros de Profesorado) }\end{array}$ \\
\hline $\begin{array}{l}\text { Segunda: } \\
\text { Trabajo con las redes } \\
\text { ( } 24 \text { meses) }\end{array}$ & $\begin{array}{l}\text { Selección de } 2 \text { redes escolares (elaboración } \\
\text { de ficha de cada Red) } \\
\text { Cuestionario abierto a equipos directivos sobre } \\
\text { la participación en la red de los centros ( } 6 \text { ítems) } \\
\text { Entrevista estructurada a coordinadores de } \\
\text { redes ( } 6 \text { ítems) } \\
\text { Cuestionario Documental semiestructurado } \\
\text { sobre la participación del centro en la red } \\
\text { Participación de investigadores en reuniones de } \\
\text { Redes }\end{array}$ & $\begin{array}{l}\text { Red A de Autismo (Castilleja) } \\
\text { Red B de Coordinación Intercentros (Osuna) } \\
\text { Equipos Directivos de } 14 \text { centros de las } 2 \text { redes } \\
\text { escolares (Director, Secretario, Jefe Estudios) } \\
\text { Coordinadores de redes } \\
\text { Profesorado de los centros participantes en las } \\
2 \text { redes escolares (Castilleja y Osuna) } \\
\text { Observación participante: notas }\end{array}$ \\
\hline $\begin{array}{l}\text { Tercera: } \\
\text { Elaborando } \\
\text { conclusiones ( } 9 \text { meses) }\end{array}$ & $\begin{array}{l}\text { Entrevistas finales informales a asesores: } \\
\text { análisis de mejora a través de la participación en } \\
\text { redes }\end{array}$ & Asesores de CEPs-Centros de Profesorado \\
\hline
\end{tabular}

La información obtenida mediante la aplicación de los cuestionarios y las entrevistas grupales de la primera fase para la obtención de una información previa de las redes escolares se han analizado en profundidad, basándonos en los trabajos del análisis del discurso y conversacional con un enfoque de tipo constructivista (Bardin, 1991; Edwards, 1997). Este método nos ha permitido examinar y explicar los datos para identificar el trabajo en red de los centros a través de un proceso de agrupación discursiva de categorías, el cual aglutina discursos comunes de los entrevistados (Ruiz-Vargas, 1996). Se han seleccionado párrafos del discurso relevantes y ajustados a nuestro análisis en el funcionamiento de las redes. Estas categorías suponen un conjunto de expectativas de los informantes en función del contexto en el que se ubican. Cada categoría dispone de información específica, no considerándose mutuamente excluyentes.

Las categorías utilizadas han surgido de manera emergente, del propio contenido del discurso obtenido en el análisis de los cuestionarios y las entrevistas en focus group a asesores y directores de Centros de Profesorado, considerándose las siguientes: inicio del trabajo y formación de la red, diseño y desarrollo de las redes/recursos, organización del trabajo en red, repercusiones en los centros y en la comunidad educativa, impacto de la participación en la red y resultados. 


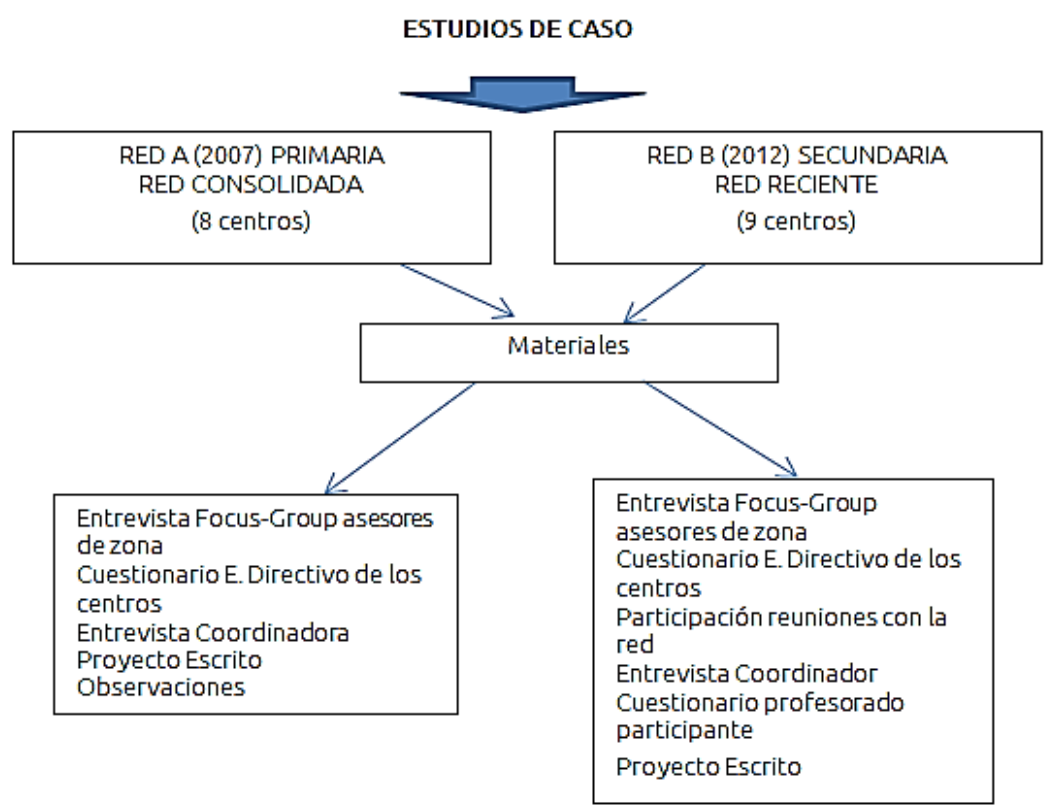

Figura 1. Estructura de la recogida de datos de los estudios de caso de la Red A y Red B

El análisis de la información es acorde con la metodología utilizada, tomando las narraciones de relevancia para comprender el significado de lo acontecido en el proceso investigado:

Toda investigación cualitativa es de hecho una investigación narrativa. Los propios informes de investigación están compuestos de narrativas, integradas de cuatro elementos: datos observacionales, relatos que los informantes cuentan, relatos escuchados por el investigador y los modelos teóricos (que a su vez son estructuras narrativas) que guían la investigación (Bolívar, Domingo y Fernández, 2001, p. 80).

\section{Análisis de datos y presentación de los resultados}

Los resultados obtenidos tras la aplicación de los instrumentos utilizados en las diferentes fases de la investigación hacen referencia a las dos redes que constituyen los dos estudios de caso:

a) Respecto a la entrevista realizada en los grupos de enfoque (focus-group) al equipo de los centros de profesorado (ver Anexo 1): el análisis de las 3 entrevistas grupales en centros de profesorado para toma de contacto con las redes ofrece los datos siguientes:

- Grupo focal 1 , integrado por director y subdirector del centro de profesorado, asesores de infantil, primaria, secundaria y necesidades educativas (atención a la diversidad), destaca que los centros que participan en redes trabajan en colaboración y en comunicación horizontal, evalúan sus necesidades y transforman las actitudes del profesorado ante los procesos formativos; asimismo, trabajan a partir del currículum común y de competencias básicas para el desarrollo de una escuela inclusiva

- Grupo focal 2, integrado por inspector de zona, director de centro de profesorado y asesores de infantil, primaria y secundaria, afirma la importancia de encontrar una motivación para el comienzo del trabajo en una red escolar, generan gran cohesión grupal en cuanto al incremento y fortalecimiento de relaciones, ofrecen oportunidades de aprendizaje a los profesionales, evitan el aislamiento docente que se produce en el aula y promueven la implicación de las familias para garantizar el proceso de aprendizaje del niño. 
- Grupo focal 3, integrado por directora de centro de profesorado y asesor de secundaria, destaca que en las redes se intercambian experiencias entre centros escolares, facilitan la comunicación y trabajo común entre los miembros de un centro y con otros centros docentes con las mismas líneas profesionales, llevando a conocer las necesidades formativas, de coordinación, seguimiento e implicación; asimismo, las redes conectan con problemas o dificultades de prácticas docentes de "colegas" de la profesión para encontrar soluciones, caminos o alternativas docentes entre todos, evitando el aislamiento.

b) Respecto al cuestionario a los asesores de redes (ver Anexo 2), los resultados del cuestionario a los 10 asesores de 3 Centros de Profesorado de la provincia de Sevilla han permitido conocer el funcionamiento de estas redes, sus repercusiones en los centros y comunidad educativa, impacto en los centros, resultados y mejoras del funcionamiento en red, los cuales concretamos a continuación:

- Las redes tienen una incidencia positiva en cada centro escolar y en su funcionamiento en temas como educación en valores, currículum, atención a la diversidad, formación del profesorado, trabajo en equipo, convivencia, desarrollo de competencias, metodología, materiales y resolución de conflictos.

- Las redes participan de modo desigual entre ellas, dando lugar en algunos centros a una participación mayoritaria, en otros de forma paulatina o participando sólo el profesorado dedicado al alumnado con necesidades educativas, de esta forma, participar en la red anima a gran parte del profesorado a colaborar, aunque no logra atraer a la totalidad del claustro, con lo que los más interesados son profesionales que atienden a alumnos con necesidades educativas.

- Las redes crean dinámicas de trabajo más colaborativas y compartidas entre los profesionales implicados en el estudio y reflexión sobre temas educativos en los que coincide el profesorado, respondiendo a la necesidad de trabajar en una estructura dinámica de funcionamiento horizontal.

- Las redes aumentan la participación de los profesores en planes de mejora de los centros, llevando a cabo una evaluación de necesidades, agrupándose para participar y cooperar en innovaciones, consensuando con más facilidad, basando sus relaciones en el respeto y preocupación mutua y desarrollando la estrategia eTwinning, es decir, hermanamientos escolares entre centros educativos para construir puentes de comunicación y aprendizaje cooperativo.

- Las redes inician el denominado ciclo de transformaciones para el diseño, desarrollo e implicación de todos en las innovaciones y mejoras, incidiendo en la organización y el funcionamiento de los centros y con repercusión en el aula.

- Las redes ayudan al desarrollo de proyectos de centro más inclusivos para mejorar la convivencia y atención al alumnado de la atención a la diversidad.

- Las redes hacen a las escuelas responsables de todos sus alumnos, diseñando procesos que apoyan estrategias didácticas de diversidad, estableciendo comunidades de apoyo a las necesidades educativas del alumnado.

- Las redes transforman las actitudes del profesorado ante procesos formativos propios y en grupos de trabajo, así como procesos de autoevaluación basados en las prácticas docentes y formación continua en función de las necesidades concretas que presenta cada centro y comunidad.

- Las redes tienen una participación desigual por parte de los distintos sectores de la Comunidad Educativa respecto a profesorado, alumnado y equipos directivos, la participación de las 
familias es escasa.

c) Respecto al cuestionario de los equipos directivos de los 17 centros que participan en las redes (ver Anexo 3), en el análisis del cuestionario de equipos directivos de los centros escolares destacan los siguientes datos:

- El trabajo colaborativo en red entre profesionales ha demostrado en este estudio que genera conocimientos compartidos, promueve la adquisición de experiencias y apoya el desarrollo profesional del profesorado más allá del aula, es un aprendizaje intencional como proceso de intercambio de conocimientos y creación de los mismos.

- Las redes impulsan la colaboración entre profesionales como principio básico para contribuir a la mejora; la participación se concibe como prioridad y forma de trabajo para superar dificultades del trabajo individual de los profesores, impulsando proyectos de trabajo compartidos con otros sectores, instituciones y entidades de la comunidad educativa.

- La red tiene una incidencia positiva en temas relacionados con la atención al alumnado, educación en valores, desarrollo del currículum, atención a la diversidad, formación del profesorado, trabajo en equipo, detección de necesidades y sistematización de procesos de trabajo.

- La red inicia en los centros un ciclo de transformaciones para el diseño, desarrollo e implicación de todos en las innovaciones y mejoras, incide en la organización y el funcionamiento de los centros y tiene una repercusión directa en las prácticas de aula.

- El trabajo colaborativo en red promueve el desarrollo de proyectos de centro más inclusivos, orientados a mejorar la convivencia, atención a los estudiantes y suponiendo un cambio de las estructuras, relaciones y prácticas educativas para integrar la diversidad del alumnado en experiencias educativas.

- La red aumenta el sentido de pertenencia a la comunidad, como espacio abierto a la participación de profesionales, organizaciones y grupos de innovación orientados a la mejora de la educación.

- La red contribuye a disminuir el aislamiento profesional, permitiendo salir del aislamiento endémico de algunas escuelas, poniendo en cuestión las estructuras rígidas y jerarquizadas del sistema educativo y activando nuevas formas de liderazgo y nuevos modos de aprendizaje en colaboración.

- El profesorado es el protagonista principal de su propio proceso de formación, en este sentido son destacables los procesos de autoevaluación basados en las prácticas docentes, y los procesos de autoformación en los que está inmerso el profesorado participante en la red.

d) Respecto a las entrevistas con los 2 coordinadores de redes (ver Anexo 4): respecto a la Red A de autismo, ésta se constituye desde la Delegación de Educación de Zona, organizando reuniones, convocando a todos los profesores de estas aulas y creando grupos de formación para profesores especialistas en autismo, fomentado a través de Centros de Profesorado. El programa de trabajo del grupo se crea a finales de curso y la elaboración del proyecto también se hace entre todos, se trabaja sobre un centro de interés y en los proyectos se incluye a la familia. El trabajo se divide en el gran grupo en tres grupos más pequeños y cada uno desarrolla un ámbito. Asimismo, reciben formación adicional en cursos de comunicación/lenguaje, psicomotricidad, habilidades sociales y emocionales. Se trabaja por proyectos a largo plazo y se evalúa la funcionalidad de los mismos a través de la práctica. Entre los programas desarrollados en esta red destaca la elaboración de un currículum de 0-6 años para este alumnado y unas carpetas de comunicación con sistema PECs (Sistema de Comunicación por Intercambio de Imágenes). El beneficio que obtienen es la reunión con otros profesores que trabajan con la misma problemática diaria en el aula, es decir, "hablar en 
el mismo lenguaje que tú entiendes".

Respecto a la Red B de coordinación inter-centros, ésta surge por el tránsito de secundaria a primero de bachillerato del alumnado de siete centros de zona rural; los resultados académicos y la capacidad del alumnado para el cambio de ciclo arroja mucha disparidad en el acceso con los problemas que esto conlleva. Esta red se constituye para acordar criterios comunes y crear un plan de tránsito entre etapas, homogeneizando ciertas prácticas en los centros de la localidad. El Servicio de Inspección convoca reuniones de zona con el equipo directivo de los centros que pertenecen al Proyecto, con sesiones de trabajo y puestas en común. Existen dos acciones formativas relacionadas con este tránsito, con lo que han creado un espacio permanente en la plataforma relacionado con este tema. La dinámica de trabajo es positiva, ya que reúne a directores y equipos directivos, se conocen personalmente y ayudan a constituir una comunidad en red profesional, ocurriendo lo mismo con profesorado, jefes de departamento, etc., de todos los centros de zona que intercambian experiencias, metodologías y criterios de evaluación. El objetivo es plasmar un documento bien elaborado y eficaz en este tránsito escolar.

e) Respecto al cuestionario al profesorado de la Red B participante en la red (ver Anexo 5): la mayoría del profesorado afirma que no se ha producido una mejora notable en los resultados académicos por la pertenencia de los centros a la red, ha sido útil para orientar información, lo que no implica una mejora inmediata, es necesario tiempo para conocer los verdaderos resultados. Se ha propuesto en esta red la evaluación por competencias; así, $70 \%$ del profesorado opina que esta evaluación no necesariamente mejora del aprendizaje por parte del alumnado debido a que los docentes invierten mucho tiempo en esta evaluación; el 30\% restante opina que "se ha producido un nuevo camino de investigación, trabajo cooperativo y resultado del aprendizaje".

Respecto a ello, el profesorado afirma en su mayoría la existencia anterior de labores de coordinación entre docentes para planificar actividades por competencias de lengua, matemáticas y sociales, pero alegan que depende de cada Departamento; algunos profesores afirman que no existe ninguna coordinación entre docentes aún, aunque creen que es necesaria.

Entre las sugerencias proponen una mayor estabilidad en cuanto a los representantes en las reuniones, atención más personalizada, pues el grupo ha sido demasiado denso para cumplir esta petición, tener en cuenta opiniones y experiencias del profesorado; llegar a acuerdos entre todos, consultar a los docentes modificaciones llevadas a cabo, envío previo de la documentación para trabajar y posteriormente llegar a puestas en común con temas prácticos, como experiencias y actividades, formación en línea para una mayor comodidad y facilidades para asistir a encuentros (horarios y proximidad).

\section{Discusión y conclusiones}

El análisis de los datos permite presentar conclusiones de esta investigación respecto a la configuración de unos indicadores que revelan cierto consenso en las opiniones de todos los participantes en estas redes educativas y se concretan en los siguientes:

- Análisis colectivo de problemas: generan motivación para el análisis colectivo de los temas de interés entre profesionales, llegando a conclusiones compartidas y participativas.

- Trabajo horizontal: se basan en el trabajo horizontal para el desarrollo de una comunicación eficaz entre compañeros, tomando decisiones de forma compartida.

- Trabajo en base a necesidades: se crean nuevas formas de trabajo basadas en el análisis de las necesidades específicas de cada uno de los centros.

- Actitudes hacia la formación: se propician cambios en las actitudes del profesorado respecto a su 
propia formación siempre en el seno de la misma y encaminada hacia la mejora de la institución escolar.

- Participación de los sectores de la comunidad: se basan en la necesidad de participación de la comunidad local para la mejora de los procesos educativos.

- Enriquecimiento profesional: se reconoce que la participación en redes escolares propicia cohesión en el grupo, incrementa la confianza, fortalece las relaciones y genera oportunidades de aprendizaje para todos los profesionales.

- Búsqueda de soluciones compartidas: se abre el centro al intercambio de experiencias entre todos los miembros de la comunidad escolar para encontrar soluciones/alternativas a las necesidades escolares de todo el alumnado entre todos y todas.

- Potenciación del sentido de pertenencia: se basan en el consenso que afirma que el futuro de la educación se encuentra en las redes de trabajo y en las prácticas docentes entre colegas, aumentando la pertenencia al grupo de trabajo del profesorado y superando el aislamiento en el aula.

Una de las conclusiones principales que arrojan estos datos se refiere a la formación del profesorado a través de la participación en estas redes escolares en los siguientes aspectos:

1) Las redes se convierten en organizaciones vivas y dinámicas que aprenden, aumentando su capacidad de formación, llegando a superar actuaciones individuales y esporádicas en el aula sin consenso.

2) Las redes mejoran la eficacia de los procesos de trabajo en el aula, superando obstáculos didácticos y organizativos.

3) En las redes se toma conciencia de la falta de formación del profesorado en distintos temas concernientes a la innovación y la mejora.

4) En las redes se toma conciencia de la capacidad para participar y modificar una realidad concreta y mejorarla, superándose barreras a través de procesos formativos.

El desarrollo profesional en red se concibe actualmente como un aprendizaje en feedback (Civís y Longás, 2015, p. 226), ya que "la consolidación de las experiencias se sustenta en la construcción de una misión común, su proyección en acciones claramente dirigidas a la resolución de las necesidades y la obtención de resultados", retroalimentando de forma positiva el trabajo en red, cada centro aporta conocimiento a la red en la que participa, trabaja en su desarrollo, y vuelve de nuevo a la red educativa para su puesta en común a través de buenas prácticas.

Podemos concluir que las redes se convierten en organizaciones que aprenden y aumentan la capacidad de mejora, superan actuaciones individuales y esporádicas, mejoran la eficacia de los procesos de cambio, superan obstáculos para la innovación, superan tomas de decisiones de tipo vertical, aumentan el sentido de pertenencia a la comunidad, toman conciencia de la capacidad para participar y mejorar las escuelas y superan barreras para avanzar. En este entorno, como señala Bolívar (2015), las escuelas necesitan aprender a crecer, desarrollarse y hacer frente al cambio

Se trata de hacer de las escuelas unas organizaciones para el aprendizaje. La mejora de los aprendizajes de los alumnos y alumnas, misión última que justifica la experiencia escolar, se hace depender de la labor conjunta de toda la escuela (p.1).

Asimismo, señalamos, junto con Santos, Lorenzo y Priegue (2013, p. 65), "la urgencia de una mejor formación para hacer frente a los desafíos que plantea el incremento de la diversidad en nuestras aulas". A partir de estos resultados nos reafirmamos en la mejora a partir de la participación de los centros en las 
redes educativas, y destacamos la función imprescindible de los asesores en la dinamización de redes locales. Los asesores ejercen, en primer lugar, una influencia directa en los procesos de cambio, propiciando proyectos globales en cada centro, desarrollando objetivos y tareas en el transcurso de los mismos y en segundo lugar instaurando, consolidando y coordinando procesos formativos en cada red de centros.

\section{Referencias}

Ainscow, M. y West, M. (2006). Improving urban school: leadership and collaboration. Buckingham: Open University Press.

Ainscow, M., Muijs, R. D. y West, M. (2006). Collaboration as a strategy for improving schools in challenging circumstances. Improving Schools, 9(3), 192-202. doi:10.1177/1365480206069014

Bardin, L. (1991). Análisis de contenido. Madrid: AKAL.

Betoret, F. D. y Artiga, A. G. (2010). Barriers perceived by teachers at work, coping strategies, sel-efficacy and burnout. The Spanish Journal of Psychology, 13(2), 637-654.

Bolívar, A. (noviembre de 2015). Construir localmente la capacidad de mejora: Liderazgo pedagógico y Comunidad Profesional. Ponencia presentada en el XIII Congreso Nacional de Investigación Educativa, Chihuahua, México.

Bolívar, A., Domingo, J. y Fernández, M. (2001). La investigación biográfico-narrativa en educación. Enfoque y metodología. Madrid: La Muralla.

Chapman, C. (2006). School improvement through external intervention? Londres: Continuum.

Chapman, C. y Fullan, M. (2007). Collaboration and partnership for equitable improvement: towards a networked learning systems? School Leadership and Management, 27(3), 205-211.

doi:10.1080/13632430701379354

Civís, M. y Longás, J. (2015). La colaboración interinstitucional como respuesta al desafío de la inclusión socioeducativa. Análisis de 4 experiencias de trabajo en red a nivel local en Cataluña. Educación XXI, 18(1), 213-236. doi:10.5944/educxx1.18.1.12318

Doménech, J. (2005). Aprendiendo en red. Aula de Innovación Educativa, 142, 51-54.

Dufour, R., Eaker, R. y Many, T. (2006). Learning by doing: a Handbook for professional learning communities at work. Bloomington, in: Solution Tree.

Elboj, C., Puigdellívol, I., Soler, M. y Valls, R. (2002). Comunidades de Aprendizaje: transformar la educación. Barcelona: Graó.

Edwards, D. (1997). Discourse and cognition. Londres: Sage

Gobierno Vasco (2011). Redes educativas. Recuperado de http://www.hezkuntza.ejgv.euskadi.eus/r432519/eu/contenidos/informacion/dig redes educativas/es redesedu/adjuntos/redes educativas c.pdf

Hadfield, M. y Chapman, C. (2009). Leading school-based networks. Gran Bretaña: Routledge. 
Ministerio de Educación del Salvador (2004-2009). Redes Escolares Efectivas. Plan de Educación 2021. Recuperado de http://www.oei.es/quipu/salvador/Redes.pdf

Muñoz, I. (2010). El trabajo en red, una apuesta de futuro. Recuperado de http://www.senderi.org/index.php?option=con contentyview=articley

Rué, J. (2005). Developing an empowered vision of teaching with networks. En W. Veugelers y M. J. O’Hair (Eds.). Network learning for educational change. Nueva York: Open University Press/McGraw-Hill.

Rué, J., Balaguer, L., Forastiello, A.M., García, A., Moreno, F., Nuñez, C. y Valls, G. (2005). El desarrollo de la profesionalidad docente mediante redes. Revista Iberoamericana sobre Calidad, Eficacia y Cambio en Educación, 3(1), 403-411.

Ruiz-Vargas, J.M. (1996). Psicología de la memoria. Madrid: Alianza Psicología

Santos, M. A., Lorenzo, M. y Priegue, D. (2013). Conectando a los profesores para el desarrollo de la interculturalidad. Educación XXI, 16(1), 63-84. doi:10.5944/educxx1.16.1.717

Vilar, J. (2008). Implicaciones éticas del trabajo en red y la acción comunitaria. Cultura y Educación, 20(3), 267-277.

Wohlstetter, P. y Smith, A. K. (2000). A different approach to systemic reform: network structures in Los Angeles. Phi Delta Kappan, 81(7), 508-515. 


\section{ANEXOS}

\section{Anexo 1. Guión entrevista focal de inicio a asesores de redes escolares}

1. Inicio del trabajo y formación de la red: cómo se desarrolla la puesta en marcha y desarrollo de las Redes, qué centros participan, quiénes, qué acogida tiene en cada centro...

2. Diseño y desarrollo de las redes: de qué necesidades parte cada uno de los centros, cuál es el contenido y los objetivos del Proyecto en Red (¿existe consenso?), ¿parten de una tradición de trabajo en equipo?, cómo se posiciona el Equipo Directivo respecto a la participación en estas Redes.

\section{Recursos: qué recursos materiales y humanos tiene cada RED.}

4. Organización del trabajo en red: cómo funcionan las redes, cómo se realiza la distribución de tareas, reuniones, organización interna en la red, en quién recae la coordinación de la red, y la coordinación en cada centro: equipos educativos, ciclos, departamentos... Cómo se desarrollan los procesos comunicativos entre los miembros de la Red, cómo se diseñan los procesos de participación en la red, cómo se abordan los conflictos.

5. Repercusiones en los centros y en la comunidad educativa: ¿Ha generado la pertenencia a la red mayor participación en cada centro?, ¿ha establecido relaciones de colaboración con otras redes?, ¿participan las familias en este Proyecto?, ¿participa algún representante municipal/Administración?, ¿participan los Equipos de Orientación Educativa en los Proyectos Redes?

6. Impacto de la participación en la red en los centros educativos: ¿Qué impacto han tenido en la vida de cada centro en general?, ¿se han mejorado aspectos relacionados con la educación en valores?, ¿en el desarrollo del currículum, la atención a la diversidad, la formación del profesorado?, ¿en qué aspectos de la Comunidad Educativa han tenido más impacto las Redes?

7. Resultados: ¿Tiene la red un Plan de seguimiento y evaluación?, ¿en qué momento/fase se encuentra actualmente cada Red (inicio, consolidación, mejora)?, ¿se están cumpliendo los objetivos o compromisos de mejora planteados inicialmente?, ¿qué obstáculos/limitaciones/problemas se han encontrado para desarrollar cada Proyecto?, ¿qué resultados destacarías de la experiencia? 


\section{Anexo 2. Cuestionario a asesores de redes}

\section{Respecto a los propios asesores}

Relación que mantienen los asesores con las Redes en relación a:

Coordinación: con quién y a quién.

Asesoramiento: qué asesoramiento y de qué tipo.

Formación: qué formación demandan, demandan certificación, qué interés tiene esta formación.

Tareas / actividades que organizan en la Red.

\section{Respecto al trabajo en la Red}

1. Incidencia de las redes en los centros: en qué se traduce la incidencia positiva en los centros, qué características tienen los centros que más/menos participan.

2. Participación de cada centro (profesorado): por qué la participación desigual.

3. Dinámicas de trabajo más colaborativas y compartidas por todos: qué estructura de trabajo tienen en las Redes, cómo se coordinan y qué funciones tienen profesores/padres/alumnos.

4. Participación en los planes de mejora de cada centro a partir de la Red: repercuten las mejoras consensuadas en la red en el funcionamiento en las prácticas de aula, horario, espacios?

\section{Anexo 3. Cuestionario sobre redes de centros escolares: equipos directivos}

1. ¿Conoce el trabajo que realiza el Grupo de Trabajo en el que participa su centro?, ¿qué opina sobre esta participación?

2. ¿Qué cambios se han producido a partir de esta participación?

3. A raíz de la participación en el Grupo de Trabajo, ¿qué mejoras se han producido en el centro?

4. A raíz de la participación del centro en el Grupo de Trabajo, ¿el profesorado está más interesado?

5. ¿El Equipo Directivo facilita la participación del centro en el Grupo de Trabajo por entender que revierte en la mejora del centro?

6. ¿El Equipo Directivo realiza propuestas de mejora en el funcionamiento del Grupo de Trabajo?

\section{Anexo 4. Entrevista a coordinadores de Red}

1. Inicio y formación de la Red (miembros que la componen, acuerdos establecidos en la Red sobre funcionamiento, formación de los subgrupos).

2. Desarrollo de la Red (comienzo del trabajo y desarrollo, participación de la familia, profesorado y equipo directivo).

3. Organización del trabajo (elección del coordinador, distribución de tareas, elaboración de materiales).

4. Formación demandada a los Centros de Profesorado (CEPs).

5. Evaluación del trabajo en la Red.

6. Comunicación entre los miembros de la Red y con otras Redes.

7. Beneficios del trabajo en Red. 


\section{Anexo 5. Cuestionario: Opinión profesorado acerca de la participación del centro en la Red}

La implicación del centro en la Red ha mejorado:

\section{A nivel de aula}

1. La identificación de las necesidades de aprendizaje que se generan en el aula.

2. El desarrollo de estrategias y metodologías innovadoras para todos.

3. La calidad de las interacciones en el proceso de enseñanza-aprendizaje.

4. La eficacia de la atención a los estudiantes NEAE.

5. La valoración del potencial de aprendizaje del alumnado en sus evaluaciones.

6. La utilización de estrategias dinamizadoras de trabajo colaborativo.

7. La resolución de conflictos entre compañeros.

8. El aprendizaje de los estudiantes por competencias.

\section{A nivel de profesorado}

9. La motivación en el desarrollo de su labor docente.

10. La colaboración, coordinación y participación entre equipos docentes.

11. La unificación de criterios para el desarrollo de competencias básicas.

12. El compromiso en los procesos de formación continua.

13. La reflexión permanente sobre la acción educativa.

14. El interés para participar en proyectos de innovación y mejora educativa.

15. El ambiente de trabajo.

\section{A nivel de centro}

16. Los cauces de participación de la comunidad educativa.

17. El desarrollo de un trabajo coordinado y compartido.

18. La inclusión educativa y social.

19. La colaboración de la administración con el equipo directivo.

20. Los procesos de planificación y gestión.

21. La colaboración y coordinación entre los equipos directivos.

22. Las relaciones con la familia. 